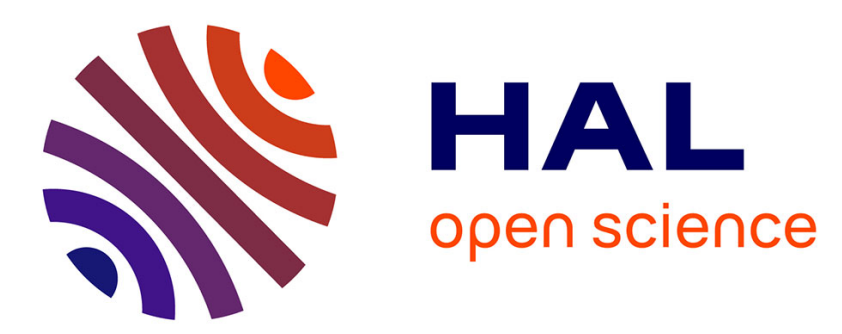

\title{
Molecular Dynamics Simulation of Configurational Properties of Complexes between $\beta$-Cyclodextrin and 2,6-Diaziadamantane in Water
}

Gerhard Zifferer, Andreas Kornherr, Udo H Brinker

\section{- To cite this version:}

Gerhard Zifferer, Andreas Kornherr, Udo H Brinker. Molecular Dynamics Simulation of Configurational Properties of Complexes between $\beta$-Cyclodextrin and 2,6-Diaziadamantane in Water. Molecular Simulation, 2008, 34 (10-15), pp.1177-1183. 10.1080/08927020802193145 . hal-00515037

\section{HAL Id: hal-00515037 \\ https://hal.science/hal-00515037}

Submitted on 4 Sep 2010

HAL is a multi-disciplinary open access archive for the deposit and dissemination of scientific research documents, whether they are published or not. The documents may come from teaching and research institutions in France or abroad, or from public or private research centers.
L'archive ouverte pluridisciplinaire HAL, est destinée au dépôt et à la diffusion de documents scientifiques de niveau recherche, publiés ou non, émanant des établissements d'enseignement et de recherche français ou étrangers, des laboratoires publics ou privés. 


\section{Molecular Simulation}

Journal of

Experimental Nanoscience

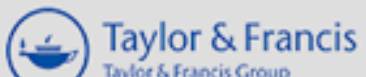

Taylor \& Francis Group

\section{Molecular Dynamics Simulation of Configurational Properties of Complexes between $\beta$-Cyclodextrin and 2,6- Diaziadamantane in Water}

\begin{tabular}{|c|c|}
\hline Journal: & Molecular Simulation/Journal of Experimental Nanoscience \\
\hline Manuscript ID: & GMOS-2008-0061.R1 \\
\hline Journal: & Molecular Simulation \\
\hline $\begin{array}{r}\text { Date Submitted by the } \\
\text { Author: }\end{array}$ & 07-Apr-2008 \\
\hline Complete List of Authors: & $\begin{array}{l}\text { Zifferer, Gerhard; University of Vienna, Institute of Physical } \\
\text { Chemistry } \\
\text { Kornherr, Andreas; Mondi Business Paper Services AG } \\
\text { Brinker, Udo; University of Vienna, Institute of Organic Chemistry }\end{array}$ \\
\hline Keywords: & $\begin{array}{l}\text { Molecular dynamics simulations, supramolecular chemistry, host- } \\
\text { guest complexes, cyclodextrin, aziadamantane }\end{array}$ \\
\hline
\end{tabular}

\section{SCHOLARONE Manuscripts}


Materials Studio special issue (word count: 3067)

\title{
RESEARCH ARTICLE
}

\section{Molecular Dynamics Simulation of Configurational Properties of Complexes between $\beta$ - Cyclodextrin and 2,6-Diaziadamantane in Water}

\author{
Gerhard Zifferer ${ }^{\mathrm{a},{ }^{*}}$, Andreas Kornherr ${ }^{\mathrm{b}}$, and Udo H. Brinker ${ }^{\mathrm{c}}$ \\ ${ }^{a}$ Institut für Physikalische Chemie, Universität Wien, Währinger Straße 42, A-1090 Wien, Austria; \\ ${ }^{b}$ Mondi Business Paper Services AG, A-3363 Ulmerfeld-Hausmening, Austria; ${ }^{c}$ Institut für \\ Organische Chemie, Universität Wien, Währinger Straße 38, A-1090 Wien, Austria
}

\begin{abstract}
Supramolecular complexes between $\beta$-cyclodextrin and 2,6-diaziadamantane in water are prepared by molecular dynamics simulations. A large number of arrangements taken from trajectories in picosecond intervals are analyzed with respect to relative orientation and compared to results obtained for 2-aziadamantane containing a single diazirine functionality only. While for the latter two stable orientations have been observed, 2,6-diaziadamantane exhibits a single favourite orientation which is similar to the energetically more preferred orientation of 2-aziadamantane. Furthermore, the influence of the guest on the shape of the host is studied in some detail.
\end{abstract}

Keywords: Molecular dynamics simulations, supramolecular chemistry, host-guest complexes, cyclodextrin, aziadamantane

\footnotetext{
*Email: gerhard.zifferer@univie.ac.at
} 
Molecular Simulation

Materials Studio special issue (word count: 3067)

RESEARCH ARTICLE

\begin{abstract}
Molecular Dynamics Simulation of Configurational Properties of Complexes between $\beta$ Cyclodextrin and 2,6-Diaziadamantane in Water
\end{abstract}

Supramolecular complexes between $\beta$-cyclodextrin and 2,6-diaziadamantane in water are prepared by molecular dynamics simulations. A large number of arrangements taken from trajectories in picosecond intervals are analyzed with respect to relative orientation and compared to results obtained for 2-aziadamantane containing a single diazirine functionality only. While for the latter two stable orientations have been observed, 2,6-diaziadamantane exhibits a single favourite orientation which is similar to the energetically more preferred orientation of 2-aziadamantane. Furthermore, the influence of the guest on the shape of the host is studied in some detail.

Keywords: Molecular dynamics simulations, supramolecular chemistry, host-guest complexes, cyclodextrin, aziadamantane 


\section{Introduction}

The preparation of inclusion complexes between good soluble host molecules and poorly soluble guest molecules is a most promising strategy to improve the solubility of compounds, e.g., for pharmaceutical formulations [1]. Furthermore, the encapsulation of precursors of reactive intermediates within host systems can influence the reaction pathways in a significant manner [2]. As a consequence the investigation of host - guest complexes has attracted much attention in recent years, above all complexes based on cyclodextrins which bind a large range of guest molecules, e.g., see [3] and references therein. Apart from experimental investigations in silico studies are most challenging to predict thermodynamic properties like binding affinities and conformational preferences of host (and guest) molecules as well as relative orientations between the host and the guest $[4,5,6]$. Recently, we studied the behaviour of complexes between $\beta$-cyclodextrin (7-CyD) and several substituted aziadamantanes in water [7]. In the present paper analogous simulations are performed for complexes between 7-CyD and 2,6-diaziadamantane (which contains two diazacyclopropen functionalities) and compared to the (monosubstituted) 2-aziadamantanes. In addition, several properties of the host molecule are calculated and the influence of guest molecules on these properties is studied.

\section{Simulation details}

Simulation details already have been given in reference [7] but are briefly reproduced in the following for convenience of the reader.

The molecular dynamics simulation was performed by use of Materials Studio® 3.0 from Accelrys Inc.: The Visualizer module served for preparing the molecules, controlling the work flow and visualization of results. Coordinates of guest and host were retrieved by use of a proper BTCL script and further code for analysis was written in FORTRAN.

First, by use of the Amorphous Cell tool a cubic box (with periodic boundaries in all directions) was prepared which contained one host (7-CyD), one guest (2,6-diaziadamantane) and 600 water molecules, see Scheme 1 . The box length was $\approx 2.72 \mathrm{~nm}$ related to a density of $1 \mathrm{~g} / \mathrm{cm}^{3}$ characteristic of ambient temperature $(298 \mathrm{~K})$. A short relaxation run under isobaricisothermal conditions at a temperature of $298 \mathrm{~K}$ left the box size unchanged confirming correct density. It should be noted that the number of water molecules was chosen in order to 
make the box large enough to avoid any interaction between a molecule and its periodic images.

Second, for several preliminary runs the guest was shifted to arbitrarily chosen positions (in two cases below and in two cases above the host) followed by an energy minimisation run in order to correct for overlaps with water molecules due to this procedure.

Third, starting from these initial systems constant temperature molecular dynamics simulations were performed using the Andersen thermostat [8] with a time step of $1 \mathrm{fs}$ applying the Verlet velocity algorithm[9]. Module Discover was the molecular dynamics engine making use of COMPASS, which is a class-II force field optimized for the simulation of condensed phases (see references in [7] for parameterization and validation). A cut-off distance of $1.25 \mathrm{~nm}$ with a spline switching function $(0.3 \mathrm{~nm}$ width) was applied for the nonbonding interactions (i.e., for Coulomb and for van der Waals interactions, the latter one being expressed by a 6-9 Lennard-Jones potential). Furthermore, charge groups were used to prevent dipoles from being artificially split when one of the atoms was inside and another was outside the atom-based cut-off. Similar to our findings for 2-aziadamantane [7] the guest entered the host within several hundred picoseconds and then remained within the host. Contrary to 2-aziadamantane where two stable configurations had been found (the diazirine group either located down (type A) or up (type B) with respect to the host, 2,6diaziadamantane exhibits a single preferred orientation due to its symmetry.

Forth, a rather long simulation run (30 ns with time step 1 fs sampled in intervals of $1000 \mathrm{fs}=1 \mathrm{ps}$ ) was performed making use of the last frame of one of the preliminary runs.

Finally, the whole trajectory was analyzed with respect to the orientation of 2,6diaziadamantane relative to 7-CyD. Furthermore, characteristic properties of 7-CyD were calculated, too. Thus, for comparison a (15 ns) run with 7-CyD in water without guest molecules was performed and analyzed in addition.

\section{Results and Discussion}

7-CyD is a cyclic heptamer of $\alpha$-D-glucose the shape of it resembling a truncated cone [10]. 2,6-Diaziadamantane is an adamantane with two diazacyclopropen functionalities at opposite atoms $\mathrm{C}_{2}$ and $\mathrm{C}_{6}$ [11]. In Scheme 2 a typical host-guest arrangement is shown using a stick model and omitting hydrogens of 7-CyD as well as water molecules. For one of the seven repeat units of 7-CyD, $\mathrm{C}$ and $\mathrm{O}$ atoms are marked by balls and labelled according to the numbering scheme used below. 
The relative orientation of host and guest may be described by the distance between their centres and the angle between the main axis of the guest and the axis of the host, i.e., the axis of the cone. The main axis of the guest is defined as the line connecting the $\mathrm{C}$ atoms bonded to $\mathrm{N}=\mathrm{N}$, i.e., $\mathrm{C}_{2}$ and $\mathrm{C}_{6}$ and the centre of gravity of the $10 \mathrm{C}$ atoms of the adamantane serves as the centre of the guest. The axis of the cone can be defined in several ways, e.g., by the smallest axis of an equivalent ellipsoid representing the actual configuration of cyclodextrin. The parameters of the equivalent ellipsoid further characterize size and shape of the host. The calculation starts with the symmetric tensor of the second kind [12]

$$
\mathbf{T}=\left(\begin{array}{lll}
t_{x x} & t_{x y} & t_{x z} \\
t_{x y} & t_{y y} & t_{y z} \\
t_{x z} & t_{y z} & t_{z z}
\end{array}\right)
$$

with components

$$
t_{x x}=\frac{1}{n} \sum_{i=1}^{n}\left(x_{i}-s_{x}\right)^{2}, t_{x y}=\frac{1}{n} \sum_{i=1}^{n}\left(x_{i}-s_{x}\right)\left(y_{i}-s_{y}\right) \text {, etc. }
$$

$\left(x_{i}, y_{i}, z_{i}\right)$ being the coordinates of the $i^{\text {th }}$ atom out of $n$ atoms constituting the molecule and $\left(s_{x}, s_{y}, s_{z}\right)$ their geometrical centre of gravity. The trace of the tensor equals the squared radius of gyration, i.e., the average of squared distances of all $n$ atoms from their common centre of gravity. Diagonalization yields eigenvectors $\mathbf{l}_{i}$ (which represent the directions of principal axes of inertia of the "equivalent ellipsoid" of the molecule in the original coordinate system) and eigenvalues $L_{i}{ }^{2}$ (which by definition are chosen as $L_{1}{ }^{2} \leq L_{2}{ }^{2} \leq L_{3}{ }^{2}$ ). The eigenvalues represent the principal components of the squared radius of gyration $s^{2}=L_{1}^{2}+L_{2}^{2}+L_{3}^{2}$ (as the trace of the matrix clearly is invariant) of individual configurations taken along the principal axes of inertia. The symmetry (or asymmetry) of conformations usually is described by shape factors $s f_{\mathrm{i}}^{*}=L_{\mathrm{i}}{ }^{2} / s^{2}$ or related quantities like the asphericity parameter

$$
\delta^{*}=1-3\left(s f_{1}^{*} \cdot s f_{2}^{*}+s f_{2}^{*} \cdot s f_{3}^{*}+s f_{3}^{*} \cdot s f_{1}^{*}\right)
$$

which ranges from 0 (spheres) to 1 (rods) or prolateness factor

$$
S^{*}=\left(3 s f_{1}^{*}-1\right)\left(3 s f_{2}^{*}-1\right)\left(3 s f_{3}^{*}-1\right)
$$

which is bounded to the interval $[-0.25,2]$ with negative values referring to predominantly oblate shapes and positive values representing prolate molecules [13,14].

In Figure $1, s^{2}$ values (based on $\mathrm{C}$ and $\mathrm{O}$ and omitting $\mathrm{H}$ atoms) of cyclodextrin snapshots taken from $15 \mathrm{~ns}$ trajectories in intervals of one picosecond are shown for 
uncomplexed (free) cyclodextrin and cyclodextrin being part of complexes with 2,6diaziadamantane. The average value reads $(0.353 \pm 0.003) \mathrm{nm}^{2}$ for the former and $(0.369 \pm 0.001) \mathrm{nm}^{2}$ the latter. (The standard deviation was calculated according to the block averaging method, e.g., see [15].) The size of the ring is slightly smaller and fluctuations are more pronounced in case of free cyclodextrin. The space required by the host obviously induces a more rigid behaviour of cyclodextrin in host-guest arrangements. As a further consequence, the shape of cyclodextrin within complexes is slightly more symmetric, $s f_{1}^{*}=0.083(0.085), \quad s f_{2}^{*}=0.432(0.398), \quad s f_{3}^{*}=0.485(0.517), \quad \delta^{*}=0.144(0.152), \quad$ and slightly more oblate $S^{*}=-0.10(-0.07)$ as compared to the free case (figures in parenthesis). This holds true for complexes with 2-aziadamantane (irrespective of the preferred orientation within the host), too: analogous investigations yield the same results as given for 2,6diaziadamantane.

For further analysis it is advantageous to transform the (Cartesian) coordinates of the host and guest into a local coordinate system defined by the principal axes of the host, the $z$ $(y, x)$ axis being identical with the smallest (medium, largest) principal axis and the larger diameter of the cone being above, the smaller (i.e., that part where the $-\mathrm{CH}_{2}-\mathrm{OH}$ groups are located) below the $x / y$-plane. The positive direction of the $x$ axis finally is defined by the $\mathrm{C}_{5}$ atom closest to this axis.

In Figure 2, 5000 cyclodextrin snapshots taken in 3 ps intervals from a 15 ns trajectory of (free) cyclodextrin in water are superimposed after transformation as described above and projected into the $x / y$ plane. Each diagram presents the positions of a specific atom with a certain colour for atoms which belong to the same repeat unit. The ellipses are drawn according to root mean square distances $\sqrt{2\left\langle L_{i}^{2}\right\rangle}(i=3, x$ axis and $i=2, y$ axis $)$. In Figure 3 equivalent diagrams are shown for snapshots taken from a $30 \mathrm{~ns}$ trajectory of 7-CyD complexes with 2,6-diaziadamantane in $6 \mathrm{ps}$ intervals. Diagrams of 7-CyD being part of complexes with 2-aziadamantane are omitted as their appearance coincides with that in Figure 3. In accordance with the behaviour of $s^{2}$ and the shape factors discussed above, fluctuations are much more pronounced in Figure 2 referring to free cyclodextrin. In all cases fluctuations are smallest for "bridging" atoms $\left(\mathrm{C}_{1(\mathrm{n})}-\mathrm{O}_{1(\mathrm{n})}-\mathrm{C}_{4(\mathrm{n}+1)}\right)$, followed by atoms constituting the ring of the glucose units, i.e., $\mathrm{C}_{2}$ and $\mathrm{C}_{3}$ (upper rim) and $\mathrm{C}_{5}$ and $\mathrm{O}_{5}$ (lower rim), oxygen $\mathrm{O}_{2}$ and $\mathrm{O}_{3}$ bound to the upper rim, then $\mathrm{C}_{6}$ bound to the lower rim and finally and most mobile, oxygen $\mathrm{O}_{6}$. 
In Figure 4(a) all $\mathrm{C}$ and $\mathrm{O}$ atoms of 7-CyD are superimposed and the positions of $\mathrm{C}_{2}$ and $\mathrm{C}_{6}$ of 2,6-diaziadamantane (black dots) are shown in addition (again 5000 guest molecules in 6 ps intervals but only 300 CyDs in 100 ps intervals, using a reduced pen width to avoid overload). Their $x$ and $y$ coordinates are located narrowly around the centre of the host. The same holds true for 2-aziadamantanes with the diazirine group located down (type A) - depicted in Figure 4(b) - while for 2-aziadamantanes with the diazirine group located up (type B) the projections are within an annulus around the centre, see Figure 4(c). The centres of the adamantanes are located within a still smaller region (not shown, but similar to the central white area in Figure 4(c). As a consequence the average angle $\alpha^{\prime}$ between the main axis of the guest (i.e., the line through $\mathrm{C}_{2}$ and $\mathrm{C}_{6}$ ) and the $z$ axis (identical with the axis of the cone) is rather small, e.g., $27.1^{\circ}$ for 2,6-diaziadamantane.

In the former paper [7] the orientation had been defined as the angle between the vector from $C_{6}$ to $C_{2}$ and the positive $z$ axis reading $\alpha=154.1^{\circ}$ for type $A(A)$ and $\alpha=43.8^{\circ}$ for type B (B) arrangements. Due to the symmetry in 2,6-diaziadamantane (termed $\mathbf{D}$ in the following) $\mathrm{C}_{6}$ and $\mathrm{C}_{2}$ are equivalent and orientation $\alpha$ and $180-\alpha$ coincide. As already being evident from Figure 4, comparing $\alpha^{\prime}=27.1$ with $\alpha^{\prime}=180^{\circ}-154.1^{\circ}=25.9^{\circ}$ and $\alpha^{\prime}=\alpha=43.8^{\circ}$ reveals that the behaviour of $\mathbf{D}$ is rather similar to that of $\mathbf{A}$ while a broader range of orientations is available for $\mathbf{B}$. The reason becomes immediately obvious from projections of the three systems into the $x / z$ plane, shown in Figure 5. The average $z$ positions of the centres of the adamantanes are almost identical (ca. $0.13-0.15 \mathrm{~nm}$ ). Either $\mathrm{C}_{2}$ or $\mathrm{C}_{6}$ of $\mathbf{D}$ (and $\mathbf{C}_{2}$ of $\mathbf{A}$ or $\mathbf{C}_{6}$ of $\mathbf{B}$, respectively) on average are located in the $x / y$ plane ( $z$ ca. $0.01 \mathrm{~nm}$ to $-0.03 \mathrm{~nm}$ ) and therefore deep in the cavity of the host. A diazirine functionality at the opposite $\mathbf{C}$ atom (i.e., one of $\mathbf{D}$ or the single one in case of $\mathbf{B}$ ) is located in the outer region of the host and, therefore, is less restricted than a diazacyclopropen group in the interior of the host. Thus, the orientation of $\mathbf{D}$ is mainly controlled by the same mechanism as in case of $\mathbf{A}$ arrangements of 2-aziadamantane. Thus, not only the average orientation angle is very similar but also the frequency distribution (based on a histogram in $1^{\circ}$ steps) of angle $\alpha^{\prime}$ as shown in Figure 6. Closer inspection of Figure 4 gives the impression that the projection points of $\mathrm{C}_{2}$ and $\mathbf{C}_{6}$ are located within an ellipse rather than within a sphere for $\mathbf{D}$ and $\mathbf{A}$ while for $\mathbf{B}$ the distribution appears quite symmetric. Thus, for a thorough investigation, in Figure 7 the frequency distribution of the (smaller) angle $\alpha^{\prime \prime}$ between the projection of the line through $\mathrm{C}_{2}$ and $\mathrm{C}_{6}$ into the $x / y$ plane and the $x$ axis is depicted. Actually, angles are uniformly distributed in case of $\mathbf{B}$ arrangements of 2-aziadamantane while in case of $\mathbf{A}$ and $\mathbf{D}$ smaller angles are 
more preferred than larger ones, obviously (mutually) induced by the non perfect symmetry of the host.

The complex remained stable over the whole simulation run of $30 \mathrm{~ns}$. Nevertheless, occasionally $\mathrm{C}_{2}$ and $\mathrm{C}_{6}$ changed their position, i.e., the guest molecule rotated in such a way that the diazirine functionality within the cavity moved up to the outer part of the host and vice versa. Clearly, this process cannot take place within the cavity. However, from time to time larger fluctuations in distance $d$ (defined as the $z$ coordinate of the centre of the guest in the transformed coordinate system) occur which allow the guest molecule to escape and in certain cases rotations occur before re-entering. As demonstrated in Figure 8, where the time evolution of angle and distance in the vicinity of such a change of orientation is shown, the whole process is completed within ca. 50-100 picoseconds.

A rough check of a certain number of arrangements using the hydrogen calculation tool of the Visualizer (which is merely based on the calculation of distances and angles within proper ranges) yields a vanishing number of arrangements with hydrogen bonds between host and guest. Thus, hydrogen bonding seems to play a minor or even no role for the stabilization of the complex; the main reason for formation and stability of the complex seems to be the less hydrophilic cavity of the host - suitable for the hydrophobic adamantane - as compared to the polar upper and lower rim of the host.

Finally, it should be noted that an analysis based on the representation of cyclodextrin by an equivalent ellipsoid is the first one as far as the authors know. Dealing with the shape, shape fluctuations and an overlay of configurations in a well-defined manner the procedure seems most promising. Further methods for structural analysis use specific intramolecular distances and torsions [3]; recently an interesting approach based on similarity maps and calculated pair distribution functions has been published [16].

\section{Conclusions}

Atomistic molecular dynamics simulations provide a valuable tool to predict a large number of interesting and important features of guest-host complexes including conformational properties and equilibrium fluctuations addressed in the present paper. Representation of the host by an equivalent ellipsoid proved most valuable for the analysis of size, shape, orientation and evolution of (relative) positions. The simulation results referring to the orientation of the guest within the host are in full accordance with experimental predictions (based on results obtained by induced circular dichroism and NMR spectroscopy) stating that the main (symmetry) axis of 2,6-diaziadamantane is roughly aligned with the 
[1] M.E. Davis and M.E. Brewster, Cyclodextrin-based pharmaceutics: past, present and future, Nat. Rev. Drug Discovery 3 (2004), pp. 1023-1035.

[2] M.G. Rosenberg and U.H. Brinker, Carbenes generated within cyclodextrins and zeolites, Adv. Phys. Org. Chem., Vol. 40, J.P. Richard, ed. Academic Press, New York, 2005, pp $1-47$.

[3] W. Chen, C. Chang, and M.K. Gilson, Calculation of cyclodextrin binding affinities: energy, entropy, and implications for drug design, Biophys. J. 87(2004), pp. 30353049.

[4] B. Lipkowitz, Applications of computational chemistry to study cyclodextrins, Chem. Rev. 98 (1998), pp. 1829-1873.

[5] A. Castro and D.A.J. Barbiric, Molecular modeling and cyclodextrins: A relationship strengthened by complexes, Curr. Org. Chem. 10 (2006), pp. 715-729

[6] H. Dodziuk, Modeling of CyDs and their complexes, in cyclodextrins and their complexes, H. Dodziuk, ed., Wiley-VCH, Weinheim, 2006, pp. 333-355.

[7] B. Sellner, G. Zifferer, A. Kornherr, D. Krois, and U.H. Brinker, Molecular dynamics simulations of $\beta$-cyclodextrin-aziadamantane complexes in water, J. Phys. Chem. B 112 (2008), pp. 710-714. 
[8] H.C. Andersen, Molecular dynamics simulations at constant pressure and/or temperature, J. Chem. Phys. 72 (1980), pp. 2384-2393.

[9] L. Verlet, Computer „experiments“ on classical fluids. I. Thermodynamical properties of Lennard-Jones molecules, Phys. Rev. 159 (1967), pp. 98-103.

[10] W. Saenger, J. Jacob, K. Gessler, T. Steiner, D. Hoffmann, H. Sanbe, K. Koizumi, S.M. Smith, and T. Takaha, Structures of the common cyclodextrins and their larger analogues-beyond the doughnut, Chem. Rev. 98 (1998), pp. 1787-1802.

[11] M.M. Bobek, D. Krois, T.H. Brehmer, G. Giester, K.B. Wiberg, and U.H. Brinker, 2.6Diaziadamantane: A single crystal X-ray diffraction study and theoretical calculations, J. Org. Chem. 68 (2003), pp. 2129-2134.

[12] K. Šolc, Shape of a random-flight chain, J. Chem. Phys. 55 (1971), pp. 335-344.

[13] J.A. Aronovitz and D.R. Nelson, Universal features of polymer shapes, J. Phys. (Paris) 47 (1986), pp. 1445-1456.

[14] G. Zifferer and A. Kornherr, Atomistic molecular-dynamics simulations of the size and shape of polyethylene in hexane at infinite dilution, J. Chem. Phys. 122 (2005), pp. 204906-1-7, and references cited therein.

[15] G. Zifferer, Monte Carlo simulations of tetrahedral chains. 1. Very long (athermal) chains by pivot algorithm, Macromolecules 23 (1990), pp. 3166-3172, and references cited therein

[16] G. Raffaini and F. Ganazzoli, Molecular dynamics study of host-guest interactions in cyclodextrins: methodology and data analysis for a comparison with solution data and the solid-state structure, J. Incl. Phenom. Macrocycl. Chem. 57 (2007), pp. 683-688.

[17] M.M. Bobek, D. Krois, and U.H. Brinker, Induced circular dichroism of cyclodextrin inclusion complexes: Examining the cavity with a bilateral probe, Org. Lett. 2 (2000), pp. 1999-2002. 


\section{Page 11 of 22}

1

2

3

4

5

6
7

8

9

10

11

12

13

14

15

16

17

18

19

20

(1)

23

24

25

26

27

29

30

31

32

33

34

35

36

37

38

39

40

41

42

43

44

45

46

47

48

49

50

51

52

53

54

55

56

57

58

59

60

http://mc.manuscriptcentral.com/tandf/jenmol 


\section{Figure Captions}

Scheme 1. Simulation box containing 600 water molecules (lines), 2,6-diaziadamantane (ball and sticks) and $\beta$ cyclodextrin (sticks). H: white, C: grey, O: red, and N: blue.

Scheme 2. Stick model of a $\beta$-cyclodextrin-2,6-aziadamantane complex omitting hydrogens of 7-CyD. C and O atoms of one of the seven repeat units are marked by balls and labelled.

Figure 1. $s^{2}$ values of 7-CyD snapshots of free cyclodextrin (black) and cyclodextrin being part of complexes with 2,6-diaziadamantane (green).

Figure 2. Overlay in the $x / y$ plane of specific $\mathrm{C}$ and $\mathrm{O}$ atoms (see Scheme 2 for labelling) of 5000 configurations of free cyclodextrin; atoms belonging to the same repeat unit are equally coloured.

Figure 3. Overlay in the $x / y$ plane of specific C and O atoms (see Scheme 2 for labelling) of 5000 configurations of cyclodextrin being part of 7-CyD-2,6-diaziadamantane complexes; atoms belonging to the same repeat unit are equally coloured.

Figure 4. Overlay in the $x / y$ plane of all $\mathrm{C}$ and $\mathrm{O}$ atoms of configurations of cyclodextrin being part of complexes with 2,6-diaziadamantane (a) and 2-aziadamantane, type-A (b) and type-B (c). Atoms belonging to the same repeat unit are equally coloured. In addition, $\mathrm{C}_{2}$ and $\mathrm{C}_{6}$ of aziadamantanes are shown in black.

Figure 5. Overlay in the $x / z$ plane of all $\mathrm{C}$ and $\mathrm{O}$ atoms of configurations of cyclodextrin being part of complexes with 2,6-diaziadamantane (a) and 2-aziadamantane of type-A (b) and type-B (c). Atoms belonging to the same repeat unit are equally coloured. In addition, $\mathrm{C}_{2}$ and $\mathrm{C}_{6}$ of aziadamantanes are shown in black and nitrogens in grey.

Figure 6. Frequency distribution of angle $\alpha^{\prime}$ in case of 2,6-diaziadamantane (squares, green), and 2aziadamanane of type-A (triangles, red) and type-B (inverted triangles, blue).

Figure 7. Frequency distribution of angle $\alpha^{\prime \prime}$; symbols as in Figure 6.

Figure 8. Time evolution of distance $d$ (a) and angle $\alpha(\mathrm{b})$ in the vicinity of a change from $\overrightarrow{\mathrm{C}_{6} \mathrm{C}_{2}}$ down to $\overrightarrow{\mathrm{C}_{6} \mathrm{C}_{2}}$ up in 7-CyD-2,6-diaziadamantane complexes. 


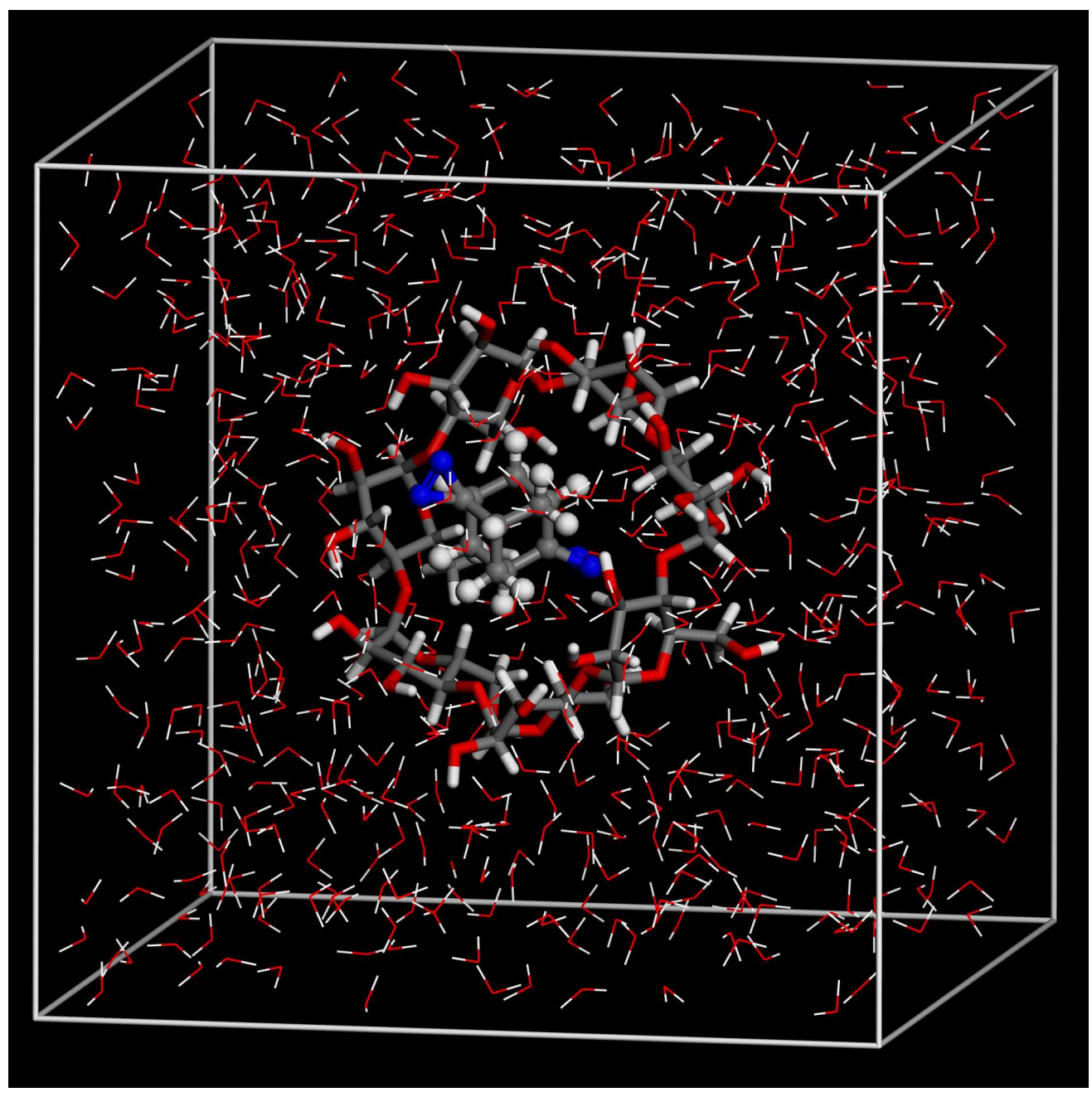

Scheme 1. Simulation box containig 600 water molecules (lines), 2,6-diaziadamantane (ball and sticks) and beta-cyclodextrin (sticks). $\mathrm{H}$ : white, $\mathrm{C}$ : grey, $\mathrm{O}$ : red, and $\mathrm{N}$ : blue. $79 \times 78 \mathrm{~mm}(600 \times 600$ DPI $)$ 


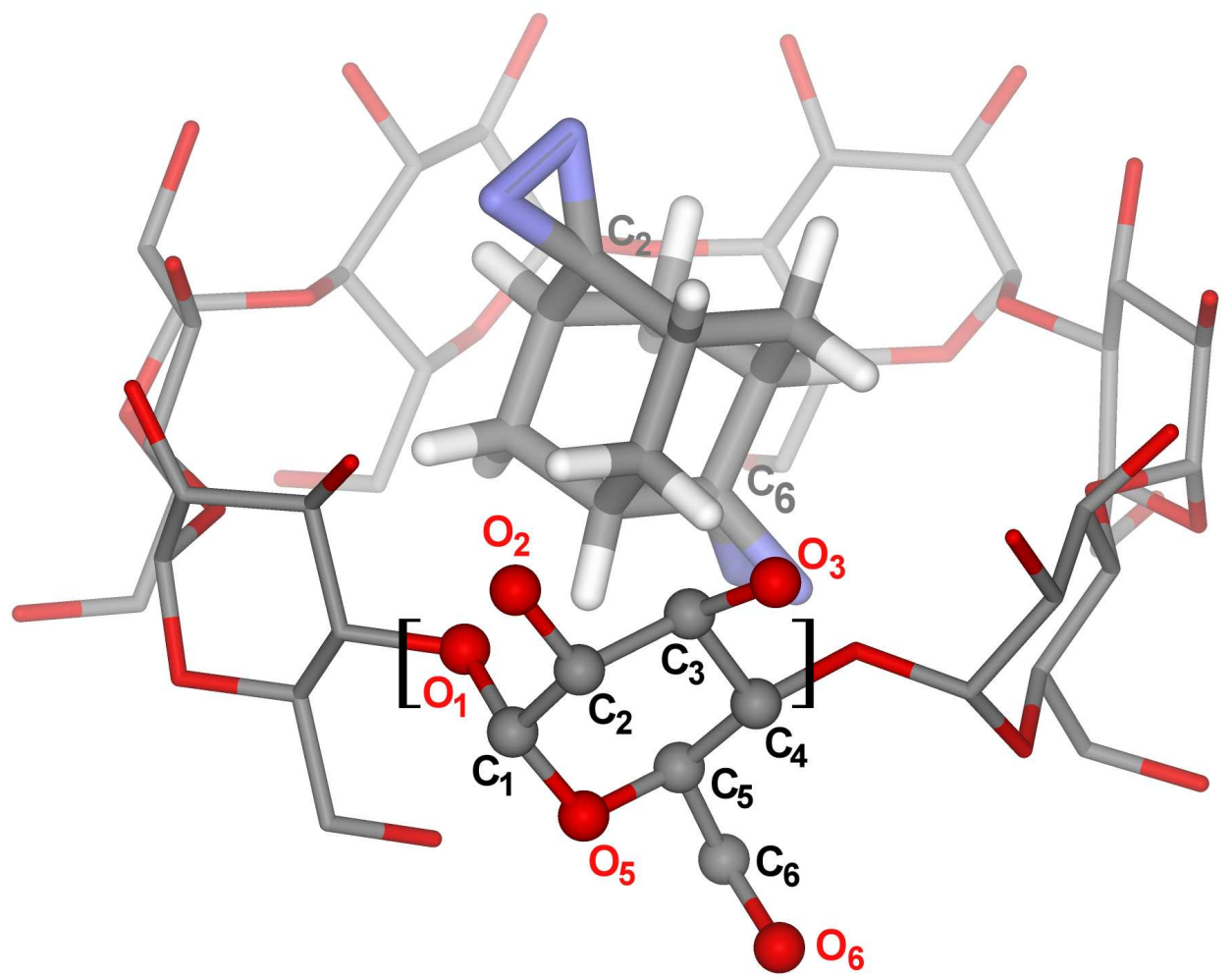

Scheme 2. Stick model of a beta-cyclodextrin - 2,6-aziadamantane complex omitting hydrogens of 7-CyD. $\mathrm{C}$ and $\mathrm{O}$ atoms of one of the seven repeat units are marked by balls and labelled. $79 \times 64 \mathrm{~mm}(600 \times 600 \mathrm{DPI})$ 
Figure 1. s2 values of 7-CyD snapshots of free cyclodextrin (black) and cyclodextrin being part of complexes with $2,6 \hat{A} \square$ diaziadamantane (green). $60 \times 45 \mathrm{~mm}(600 \times 600 \mathrm{DPI})$ 

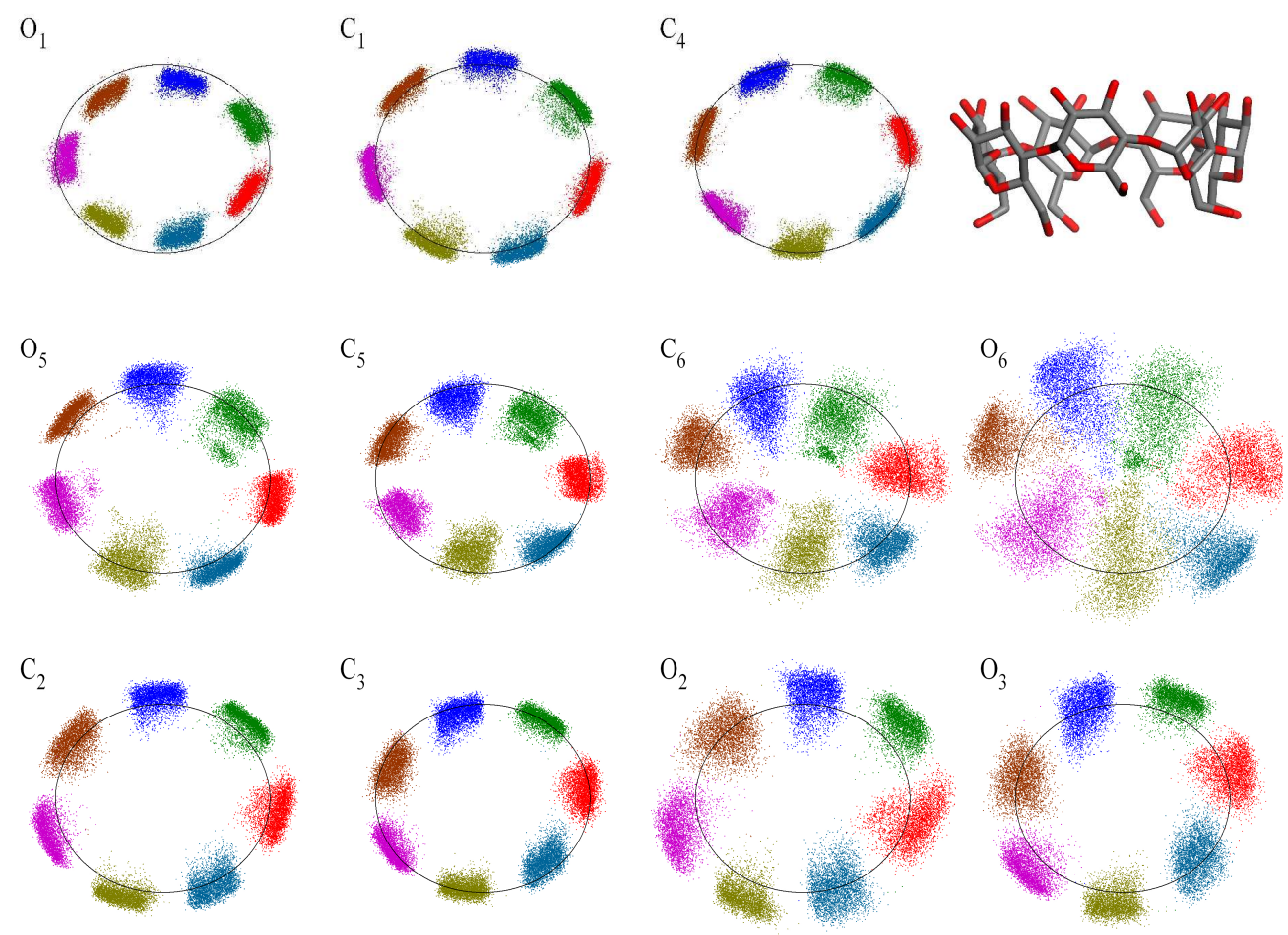

Figure 2. Overlay in the $x / y$ plane of specific $C$ and $O$ atoms (see Scheme 2 for labelling) of 5000 configurations of free cyclodextrin; atoms belonging to the same repeat unit are equally coloured. $160 \times 120 \mathrm{~mm}(600 \times 600 \mathrm{DPI})$ 

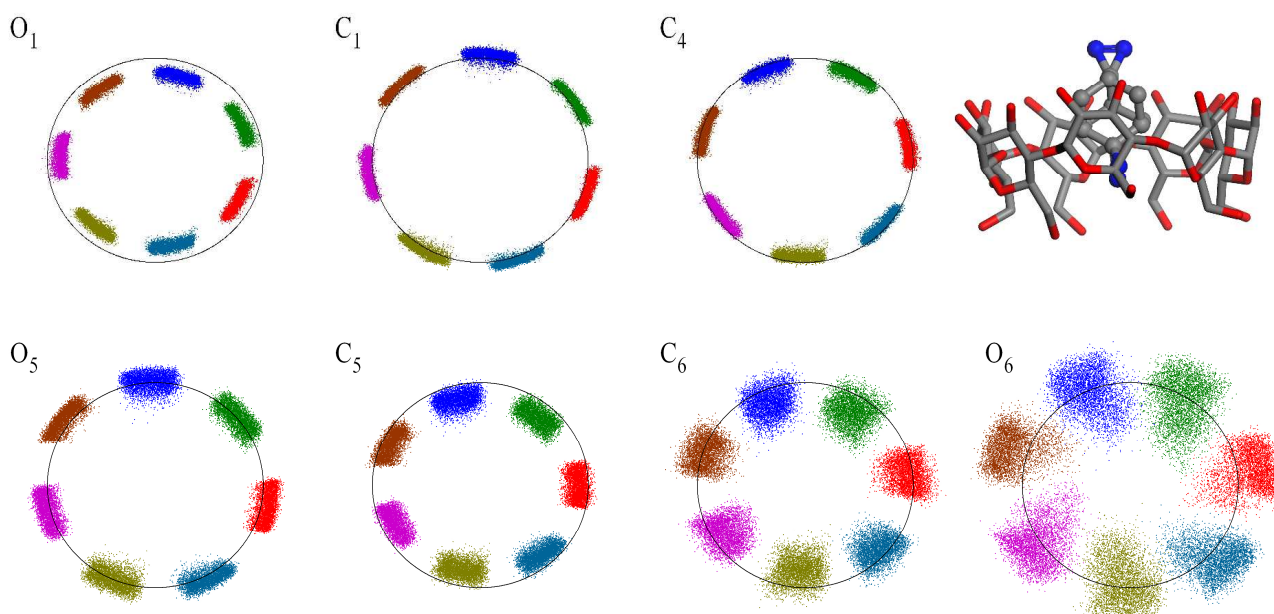

$\mathrm{C}_{5}$
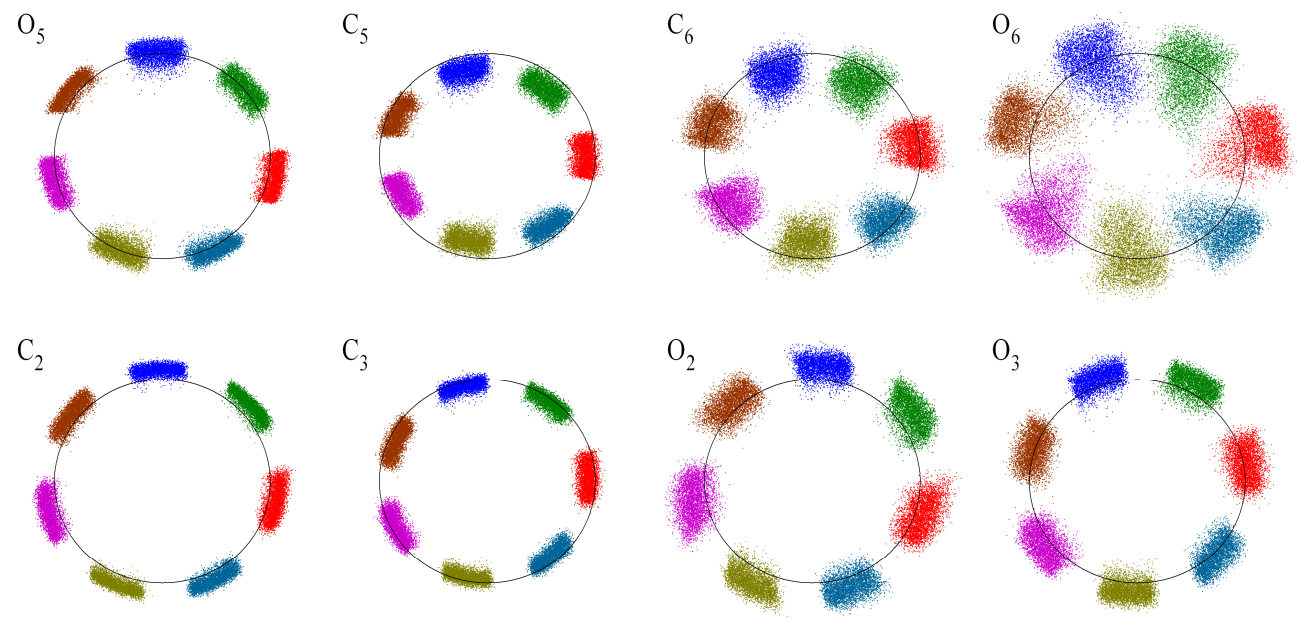

Figure 3. Overlay in the $x / y$ plane of specific $C$ and $O$ atoms (see Scheme 2 for labelling) of 5000 configurations of cyclodextrin being part of 7-CyDÂn 2,6-diaziadamantane complexes; atoms belonging to the same repeat unit are equally coloured. $160 \times 120 \mathrm{~mm}(600 \times 600 \mathrm{DPI})$ 


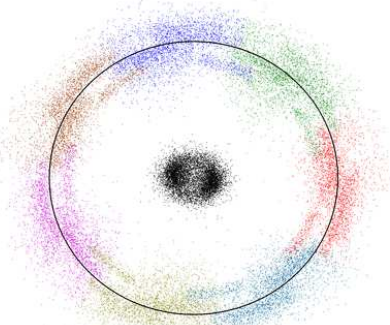

(a)

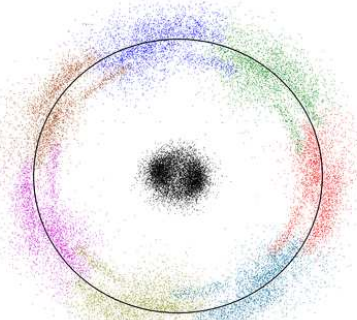

(b)

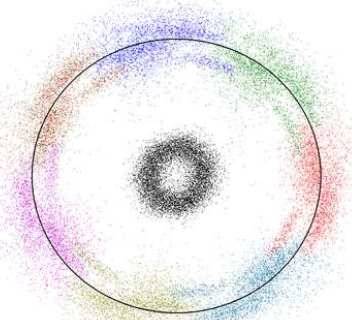

(c)

Figure4. Overlay in the $\mathrm{x} / \mathrm{y}$ plane of all $\mathrm{C}$ and $\mathrm{O}$ atoms of configurations of cyclodextrin being part of complexes with 2,6-diaziadamantane (a) and 2-aziadamantane, type-A (b) and type-B (c). Atoms belonging to the same repeat unit are equally coloured. In addition, C2 and C6 of aziadamantenes are shown in black.

\section{$53 \times 17 \mathrm{~mm}(600 \times 600 \mathrm{DPI})$}


Figure 5. Overlay in the $x / z$ plane of all $C$ and $O$ atoms of configurations of cyclodextrin being part of complexes with 2,6-diaziadamantane (a) and 2-aziadamantane of type-A (b) and type-B (c). Atoms belonging to the same repeat unit are equally coloured. In addition, C2 and C6 of aziadamantenes are shown in black and nitrogens in grey.

$47 \times 14 \mathrm{~mm}(600 \times 600$ DPI $)$ 
Figure 6. Frequency distribution of angle in case of 2,6-diaziadamantane (squares, green), and 2-aziadamanane of type-A (triangles, red) and type-B (inverted triangles, blue).

$60 \times 45 \mathrm{~mm}(600 \times 600 \mathrm{DPI})$ 
Figure 7. Frequency distribution of angle alpha-dash-dash ; symbols as in Figure 6. $60 \times 45 \mathrm{~mm}(600 \times 600 \mathrm{DPI})$ 
Page 22 of 22

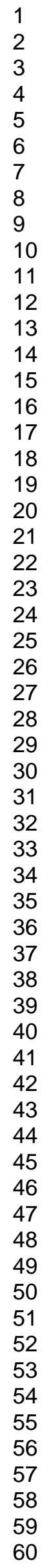
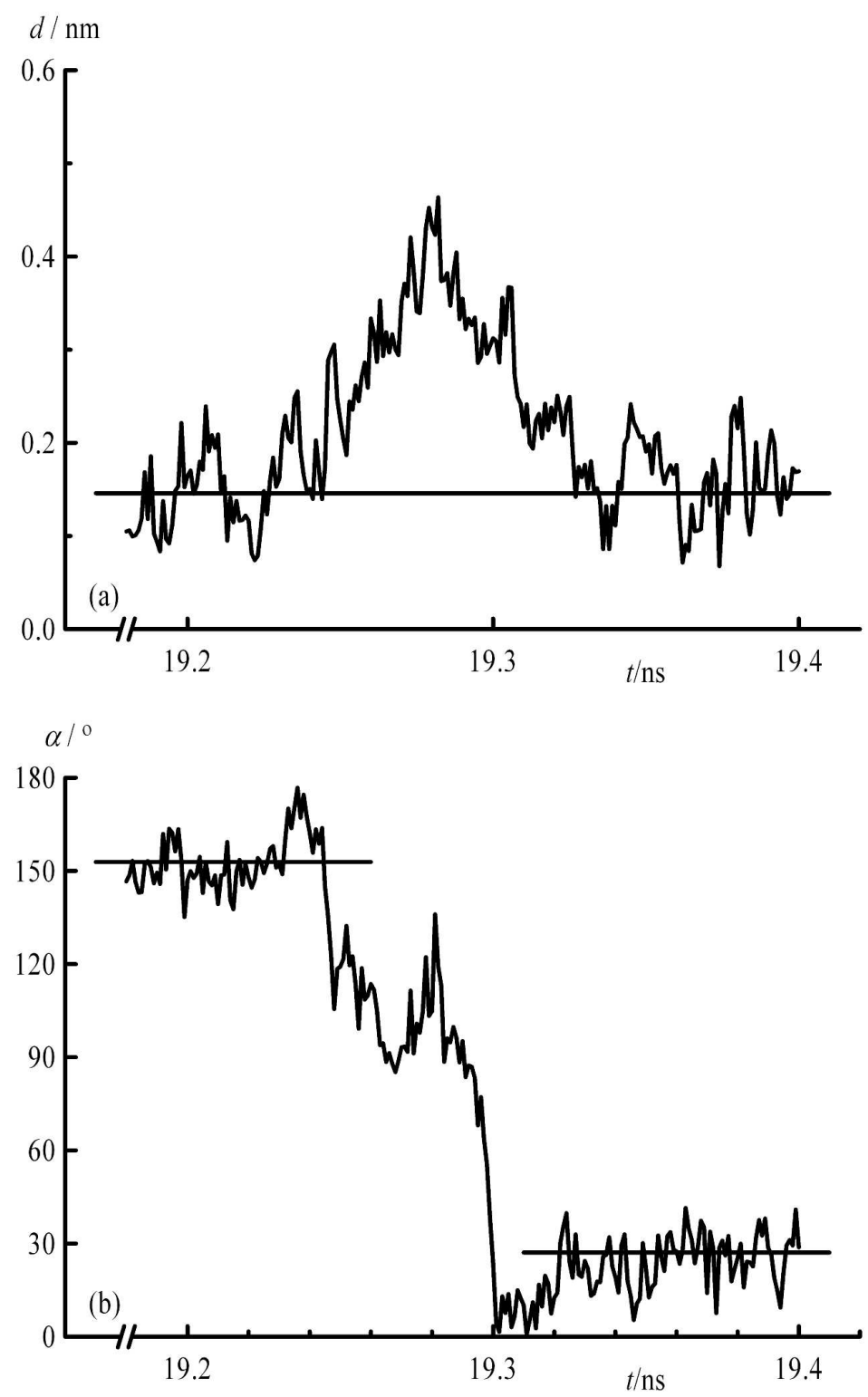

Figure 8. Time evolution of distance $d(a)$ and angle alpha (b) in the vicinity of a change from down to up in 7-CyDÂ 2,6-diziadamantane complexes.

$120 \times 180 \mathrm{~mm}(600 \times 600 \mathrm{DPI})$

http://mc.manuscriptcentral.com/tandf/jenmol 